\title{
Clinical profile of Spondarthritis in females as compared to male patients with reference to HLAB27: A single center study from Nepal
}

\author{
Binit Vaidya, ${ }^{{ }^{*}}$ Shweta Nakarmi, ${ }^{a}$ Pujan Batajoo ${ }^{\mathrm{b}}$ \\ ${ }^{a}$ Alka Hospital, Jawalakhel, Kathmandu, Nepal \\ ${ }^{b}$ Nepal Medical College, Kathmandu, Nepal
}

\author{
Accepted on \\ November $26^{\text {th }}, 2012$ \\ DOI Name \\ 10.3126/jaim.v2i1.7628

\section{Keywords} \\ Ankylosing, Arthritis, HLA B27, spondylarthritis, \\ spondylarthropathy \\ Citation \\ Vaidya B, Nakarmi S, Batajoo P. Clinical profile of \\ Spondarthritis in females as compared to male \\ patients with reference to HLAB27: A single center \\ study from Nepal. Journal of Advances in Internal \\ Medicine 2013;02(01):3-5.
}

\begin{abstract}
Background and Aims-To study the differences in clinical parameters between male and female patients with spondarthritis ( $\mathrm{SpA}$ ) in Nepalese population; to study the prevalence of HLA B27 phenotype in Nepalese patients with SpA and to study the differences in clinical parameters in relation to HLA B27 phenotype.
\end{abstract}

Methods- This is cross sectional observational study of 102 patients diagnosed with SpA attending rheumatology clinic at Alka Hospital, Nepal from September 2011 to February 2012. Electronic clinical registry was maintained and HLA B27 was performed by flowcytometry.

Results- A total of 102 patients with SpA were enrolled in the study with male to female ratio of 1.8:1. The mean age of male patients was $6 \pm 3$ years lower than that female patients $(p=0.05)$. There were no other statistically significant differences in clinical parameters or prevalence of HLA B27 in both the genders. Of the total patients, 52.9\% were HLA B27 positive. Presence of HLA B27 was associated with early presentation by $7.1 \pm 2.8$ years and the occurrence of inflammatory spinal pain $(P<0.001)$. Statistically significant association of HLA B27 with other clinical manifestations of SpA were not seen.

Conclusions- HLA B27 was present in 52.9\% of patients with diagnosed SpA. The presence of HLA B27 was associated with earlier presentation of disease and occurrence of inflammatory spinal pain. However, there was no gender difference in clinical manifestation of SpA or prevalence of HLAB27 in our patients except for early age at presentation in male patients.

\section{INTRODUCTION}

Spondarthropathies (SpA) are a group of conditions affecting the spine and peripheral joints, which cluster in families and are, linked to certain type 1 HLA antigens. These disorders include ankylosing spondylitis, reactive arthritis, psoriatic arthritis and spondylitis, enteropathy associated arthritis and spondylitis, juvenile-onset spondyloarthritis, and undifferentiated spondyloarthritis. ${ }^{1}$ The disease usually begins in the second or third decade of life with male to female prevalence between $3: 1$ and 5:1. The symptoms of the disease are usually first noticed in late adolescence or early adulthood..$^{2,3}$ The course of the disease is extremely variable, ranging from the individual with mild stiffness and radiographically equivocal sacroilietis to the patient with a totally fused spine and severe bilateral hip arthritis, accompanied by severe peripheral arthritis and extra articular manifestations. In a typical severe untreated case with progression of the spondylitis to syndesmophyte formation, the patient's posture undergoes characteristic changes, with obliterated lumbar lordosis, buttock atrophy, and accentuated thoracic kyphosis. The most common extra articular manifestation is acute anterior uveitis, which occurs in $40 \%$ of patients. Up to $60 \%$ of patients have inflammation in the colon or ileum. This is usually asymptomatic, but frank inflammatory bowel disease (IBD) occurs in $5-10 \%$ of patients with Ankylosing Spondylitis (AS). ${ }^{4}$

The presence of HLA B27 is neither necessary nor sufficient for the diagnosis. However, in accord with Bayes' theorem, ${ }^{5}$ the presence or absence of HLA B27 greatly increases or decreases, respectively, the probability of $A S$ in patients with equivocal clinical findings lacking radiographic abnormalities. Moreover, the absence of HLA B27 in a typical case of AS significantly increases the probability of coexistent IBD or psoriasis. However, the inclusion of HLA B27 in theof Assessment of SpondyloArthritis international Society(ASAS) criteria in 2009 highlights its importance in the diagnosis of SpA. ${ }^{6}$

We intend to study the differences in clinical parameters of patients with $\mathrm{SpA}$ in relation to gender and presence and absence of HLA B27 phenotype. Also, we intend to evaluate the prevalence of the B27 phenotype in SpA patients.

\section{METHODS}

Across sectional observational study was conducted in which consecutive patients visiting rheumatology out-patient department (OPD) at Alka Hospital, Kathmandu, a tertiary care center from September 2011 to February 2012 were included. Both newly diagnosed as well as established cases;aged 15 years or more were enrolled in the study. Diagnosis of $\mathrm{SpA}$ was made on the basis of clinical findings using the modified New York criteria ${ }^{7}$ and ASAS criteria. ${ }^{6}$ Inflammatory back pain was diagnosed using the Indian Rheumatology Association consensus statement on diagnosis and management of SpA. ${ }^{8}$ HLA B27 testing was done using flowcytometrymethod.Patients with other autoimmune overlaps like Rheumatoid Arthritis, Systemic Lupus Erythrematosus, Reactive arthritis, IBD and psoriatic arthritis were excluded from the study.

\footnotetext{
* Corresponding author

Dr. Binit Vaidya

$M B B S, M D, F A C R$

Medicine Department, Alka Hospital, Jawalakhel,

Kathmandu, Nepal

Email: drbinitvaidya@yahoo.com
} 
Clinical details, radiological findings and serology reports were recorded in a predesigned excel sheet which included age, gender of patients, duration since symptom was first noticed, joints involved, morning stiffness, extraarticular features like skin, eyes, renal, cardiac or pulmonary involvement, and radiological findings, c-reactive protein(CRP) and HLA B27.

The estimated sample size was 70 for the study power of $95 \%$ and a significance level of 0.05. All analyses were performed using SPSS 19. Chi square test was used to identify the difference in clinical parameters of patients. Independent sample t-test was used to test the difference in continuous variables. Fisher exact test was used to calculate $p$ value to assess the correlation between categorical variables.

\section{RESULTS}

A total of 102 patients were enrolled in the study. Mean age of our patients was $39.1 \pm 14.9$ (15-75) years. Among them $64.7 \%$ were males making a male to female ratio of 1.8:1. The median disease duration was 30 months.

\section{Difference in male and female patients:}

The mean age and disease duration of male and female patients with SpA are shown in table 1.

Table 1. Difference in age and disease duration in relation to gender.

\begin{tabular}{lccc}
\hline Variable & Male $(\boldsymbol{n}=66)$ & Female $(\boldsymbol{n}=\mathbf{3 6})$ & P value \\
\hline Age & $37.0(14.5)$ & $43.9(14.9)$ & 0.05 \\
Duration of disease & $48.7(42.8)$ & $40.3(54.4)$ & 0.39 \\
\hline
\end{tabular}

It was seen that the mean age of male patients were lower than female patients by average of $6 \pm$ 3years $[p=0.05]$. However there was no difference in disease duration of these patients. There was no statistically significant difference in clinical parameters of male and female patients in terms of peripheral or axial involvement, presence or absence of sacroileitis or extra-articular manifestations. Also, there was no statistically significant difference in the prevalence of HLA B27 phenotype in male and female patients with SpA (Table 2).

Table 2. Difference in clinical parameters between male and female patients

\begin{tabular}{lccc}
\hline Parameter & Male & Female & P value \\
\hline Small joint & 17 & 12 & 0.60 \\
Large joint & 47 & 25 & 0.20 \\
Sacroilietis & 8 & 4 & 0.96 \\
InflammatorySpinalPain & 39 & 16 & 0.21 \\
Deformities & 4 & 4 & 0.66 \\
HLA B27 positive & 38 & 16 & 0.22 \\
\hline
\end{tabular}

\section{Relationship with HLA B27 phenotype:}

Fifty four(52.9\%) of patients were HLA B27 positive (38 males and 16 females; $p=0.22$ ). The mean age and disease duration of patients with or without HLA B27 phenotype are shown in table 3:
Table 3. Relationship of HLA B27 with age and duration of disease

\begin{tabular}{lrrr}
\hline \multicolumn{1}{c}{ variables } & \multicolumn{2}{c}{ HLA B27 } & \\
& $\begin{array}{l}\text { Positive } \\
(\boldsymbol{n}=54)\end{array}$ & $\begin{array}{c}\text { Negative } \\
(\boldsymbol{n}=48)\end{array}$ & P value \\
\hline Age (years) & $35.7(14.6)$ & $42.9(14.4)$ & 0.01 \\
Duration of disease & $44.6(47.3)$ & $47.0(47.4)$ & 0.79 \\
\hline
\end{tabular}

It was seen that patients with HLA B27 phenotype presented 7.1 22.8 years earlier than HLA B27 negative patients $[p=0.01]$. When associations were tested for HLA B27 phenotype with various clinical parameters, it was seen that the presence of HLA B27 showed statistically significant positive association $[p=<0.001]$ with the occurrence of inflammatory spinal pain and showed statistically significant negative association with muscle pains $[p=0.02]$. Overall 55 patients had inflammatory spinal pain(ISP) of whom $78.1 \%$ were found to be B27 positive. However, it did not show any statistically significant association with other clinical parameters like extraarticular manifestations, deformities, sacroileitisetc (table4).

Table 4. Relationship of HLA B27 with other clinical parameters.

\begin{tabular}{lccc}
\hline \multicolumn{1}{c}{ Parameters } & $\begin{array}{c}\text { HLA B27 } \\
\text { positive }\end{array}$ & $\begin{array}{c}\text { HLA B27 } \\
\text { negative }\end{array}$ & $\begin{array}{c}\text { P value } \\
\text { (from Fisher test) }\end{array}$ \\
\hline Small joint & 16 & 13 & 0.54 \\
Large joint & 38 & 34 & 0.99 \\
Sacroilietis & 20 & 13 & 0.22 \\
Inflammatory Spinal Pain & 43 & 12 & $<0.001$ \\
Deformities & 6 & 2 & 0.30 \\
Muscle pains & 10 & 20 & 0.02 \\
\hline
\end{tabular}

\section{DISCUSSION}

Spondarthritis is an inflammatory rheumatic disease predominantly involving the spine and large joints. The disease is more prevalent in males as compared to females with ratio of 2.6 to $5: 1$. $^{2,3}$ The same pattern was seen in our patient population though we have only enrolled those attending rheumatology services and not surveyed the general population. However the resemblance in gender ratio would represent similar interpretation of our findings in the general population.

The median age of our patients was 36.5 years and it was seen that male patients were approximately six years younger than female patients and the difference was statistically significant. This could mean that either male patients had severe disease or they were more aware of the disease symptoms and came for early medical attention. However as the total disease duration were not different in males and females, we would interpret it as severe disease in male patients in same period of time. The pattern of joint involvement, prevalence of peripheral joint involvement, extra-articular manifestations were not different in male and female patients in contrast to prior studies. Two studies have shown association of female gender with extra-axial involvement and extra-articular involvement. ${ }^{2,9}$ Similarly, the prevalence of HLA B27 phenotype was not different in male and female patients with SpA in our study whereas prior Indian study showed it to be more prevalent in males (81.8\%) as compared to females. ${ }^{10}$ 
Analysis in relation with HLA B27 phenotype revealed few peculiar associations in our study. This phenotype was positive in $52.9 \%$ of patients in our study. The prevalence was around $69 \%$ in a study of SpA in Brazilian patients, ${ }^{11} 82.6 \%$ in Chinese patients, ${ }^{12} 73.4 \%$ in Iranian patients ${ }^{9}$ and $43.6 \%$ in Indian patients. ${ }^{10}$ It was seen that presence of B27 was associated with younger age at presentation (by approximately 7 years) and again as the duration of disease was not significantly different, it could be deduced that presence of B27 phenotype was associated with severe disease manifestation in these patients. Also, presence of B27 was significantly associated with inflammatory spinal pain $[p=<0.001, \rho=0.55]$ in patients with SpA. It was not associated with any other clinical parameters including pattern of joint involvement or extra-articular features. However, the presence of B27 was inversely related to occurrence of muscle pains in these patients. Epidemiological study on SpA from Korea has shown association of HLAB27 with younger age of onset (by 5.3 years), with more uveitis and with hip joint involvement. Our results agree with the younger age in B27 patients but do not confirm the increased association of uveitis and joint involvement pattern with B27. ${ }^{13}$ Similarly, Indian study shows HLA B27 to be more prevalent in younger patients with SpA and agrees to the aggressive nature of disease in these patients. ${ }^{10} \mathrm{~A}$ recent 3 year prospective cohort study involving 25 centers in France has shown a prevalence of HLA B27 to be $61.5 \%$ and its presence was associated with younger age at onset of IBP and less delay in diagnosis. ${ }^{14}$
Present study included only those patients who attended rheumatology clinic and thus might not represent the true prevalence of HLA B27 positivity in the population. The results might also have been affected by differences in healthcare seeking behavior and education in male and female population of our country.

\section{CONCLUSION}

It can be concluded from our study that in Nepalese population with SpA, male and female patients did not differ significantly in terms of clinical presentation, disease duration, pattern of joint involvement and HLA B27 positivity. However, it was seen that male patient presented early or were diagnosed earlier than the female patients which might mean earlier and more problematic disease manifestation in male gender. The presence of HLA B27 phenotype was associated with earlier age of presentation and diagnosis of SpA. It was also associated more with complaints of inflammatory spinal pains than HLA B27 negative patients. Thus, HLA B27 testing can still be useful in predicting the presence, nature and severity of SpA in our population.

\section{ACKNOWLEDGEMENT}

Dr Nija Rajbhandari, MBBS for helping in manuscript preparation.

\section{REFERENCES}

1. Dougados $M$, Baeten D. Spondyloarthritis. Lancet 2011;377:2127-37.

2. Aggarwal R, Malaviya AN. Clinical characteristics of patients with ankylosing spondylitis in India. Clin Rheumatol 2009;28:1199-205.

3 Kennedy LG, Will R, Calin A. Sex ratio in the spondyloarthropathies and its relationship to phenotypic expression, mode of inheritance and age at onset. J Rheumatol 1993;20:1900-4.

4. Collantes E, Zarco P, Muñoz E, et al. Disease pattern of spondyloarthropathies in Spain: description of the first national registry (REGISPONSER)-extended report. Rheumatology (Oxford) 2007;46:1309-15.

5. Bayes T. An Essay Toward Solving a Problem in the Doctrine of Chances. Philosophical Transactions of the Royal Society of London 1764;53:370-418.

6. Rudwaleit $M$, Landewé $R$, van der Heijde $D$, et al. The development of Assessment of SpondyloArthritis international Society classification criteria for axial spondyloarthritis (Part I and II): classification of paper patients by expert opinion including uncertainty appraisal. Ann Rheum Dis 2009;68:770-83.

7. van der Linden S, Valkenburg HA, Cats A. Evaluation of diagnostic criteria for ankylosingspondylitis.A proposal for modification of the New York criteria. Arthritis Rheum 1984;27:361-8.

8. Malaviya AN, Shankar S, Arya V, et al. Indian Rheumatology Association consensus statement on the diagnosis and treatment of axial spondyloarthropathies. Indian Journal of Rheumatology 2010;5:16-34.

9. Nazarinia MA, Ghaffarpasand F, Heiran HR. Pattern of ankylosing spondylitis in an Iranian population of 98 patients. Mod Rheumatol 2009;19:309-15.

10. Sonkar GK, Usha. Role of HLA B27 in diagnosis of seronegative spondyloarthropathies. Indian J Pathol Microbiol 2007;50:908-13.

11. Sampaio-Barros PD. Epidemiology of spondyloarthritis in Brazil. Am J Med Sci 2011;341:287-8.

12. Liao $\mathrm{ZT}$, Pan $\mathrm{YF}$, Huang $\mathrm{JL}$, et al. An epidemiological survey of low back pain and axial spondyloarthritis in a Chinese Han population. Scand J Rheumatol 2009;38:455-9.

13. Kim TJ, Kim TH. Clinical spectrum of ankylosing spondylitis in Korea. Joint Bone Spine 2010;77:235-40.

14. Chung HY, Machado P, Désirée van der Heijde, et al. HLA-B27 positive patients differ from HLA-B27 negative patients in clinical presentation and imaging: results from the DESIR cohort of patients with recent onset axial spondyloarthritis. Ann Rheum Dis 2011;70:1930-6. 